\title{
Right-handed one day, right-handed the next day?
}

Short-term test-retest reliability of infant handedness

\author{
Jacqueline Fagard ${ }^{* 1} \&$ Daniela Corbetta ${ }^{2}$ \\ Eszter Somogyi ${ }^{1}$, Anna Safar $^{1}$ \& Cyann Bernard ${ }^{1}$
}

${ }^{*}$ Corresponding author

${ }^{1}$ Integrative Neuroscience Cognitive Center, University Paris Descartes CNRS

${ }^{2}$ Department of Psychology, The University of Tennessee Knoxville, TN, USA 


\begin{abstract}
Although a population bias toward right-hand preference is observed at the early stage of grasping, it is well known that at the group level hand preference is not very strong in young infants and fluctuates until the second year and even beyond. Given these fluctuations, one can wonder whether testing a young infant on a single occasion gives reliable results of its handedness. Several studies have investigated fluctuations over months or weeks but very few studies have evaluated short-term test-retest reliability. This was the goal of this study in which 21 infants aged 9 to 15 months were tested for handedness every day or with exceptionally two days between successive sessions for a total of 5 sessions. At all sessions, the infants were given the same classical handedness baby test in which 8 to 16 objects (mean: 11.25) were presented at the midline. Their handedness index $(\mathrm{HI})$ and their category of handedness derived from their $\mathrm{HI}$ were compared across sessions.
\end{abstract}

The results show that at the group level the distribution of handedness does not differ significantly across the five sessions. At the individual level, only $19 \%$ of infants were categorized as right-handed at all five sessions while $47.6 \%$ were consistent in using more the right hand than the left across the five sessions. When adding one infant whose consistency was to use more her left-hand across sessions, the overall consistency in direction of hand preference was $52.4 \%$. Most of the fluctuations across sessions occurred between being right-handed and non-lateralized or between being left-handed and nonlateralized, rather than between being right-handed and left-handed. In addition, when the results of the first sessions were compared with the results from all other sessions, changes in category of handedness occurred only for one infant (4.8\%). Most infants who changed category, changed from being lateralized to not being lateralized or the reverse. More than half of the infants remained in the same category.

These results indicate that testing handedness at the ages of 9 to 15 months gives fairly reliable results in terms of direction of hand preference, but less so in terms of its degree.

Keywords: handedness, infant, short-term test-retest evaluation 


\section{Introduction}

Some research indicates that handedness is a very precocious phenomenon, starting before birth. Indeed, at 15 weeks of gestation, $87 \%$ of fetuses appear to prefer to suck their right thumb (Hepper, Shahidullah, \& White, 1991; however, for different results, see de Vries, Wimmers, Ververs, Hopkins, Savelsbergh, \& van Geijn, 2001), an early hand preference that was shown to predict fairly well the infant's handedness 12 years later (Hepper, Wells, \& Lynch, 2005). However, the age at which the distribution of handedness at the population level is comparable to adults remains unclear: even though the percentage of right-handers outnumbers deeply that of left-handers from as early as handedness can be evaluated, the percentage of non-lateralized infants is often found to be the largest, in any case much larger than in adults (Corbetta \& Thelen, 1999; Fagard, 1998; Nelson, Campbell, \& Michel, 2013). In addition, handedness goes through many fluctuations during infancy (Corbetta \& Thelen, 1999; Fagard, 1998; Fagard \& Lockman, 2005; Jacobsohn et al., 2014), and is not stable until 3 years of age or even later (McManus, 2002; Gesell \& Ames, 1947). For instance, longitudinal studies have shown that only a small percentage of infants, about $30-35 \%$, could be consistently classified as right-handed (or more seldom, left-handed) across successive sessions separated by a few weeks (e.g. Nelson, Campbell, \& Michel, 2014). Given such large fluctuations in hand use in early development, how can we be sure that the handedness of a infant evaluated on one day, will remain the same if the same infant is evaluated again a few hours later or the next day? This raises the critical question of how representative and valid is the handedness index obtained at a single session in early development. The goal of the present study was to address this question of day-to-day reliability of infants' measure of handedness over repeated sessions within a week.

Methodological discrepancies have been highlighted to explain the variability often found in infant handedness. These include the number of trials, the number of sessions, the formula used to calculate a handedness index, and the criteria used for categorizing handedness groups from this index (Campbell, Marcinowski, Latta, \& Michel, 2015; Fagard, Margules, Lopez, \& Granjon, 2016). Two other reasons may explain the variability of infant handedness even when using the same methodology. One is the task on which hand preference is evaluated. Infants' handedness is typically assessed based on the hand chosen for reaching for and grasping objects because these are the first manual skills they master. But object grasping has been shown to be very sensitive to task characteristics in terms of precision (Fagard \& Lockman, 2005) and object spatial position in older children (Gabbard \& Helbig, 2004; Gabbard \& Rabb, 2000; Gabbard, Rabb Helbig, \& Gentry, 2001; Leconte \& Fagard, 2004, 2006) and in adults (Bryden \& Roy, 2006). In adults, it has been shown that grasping simple objects does not elicit strong hand preference. For instance, object grasping induces a stronger expression of hand preference when the object is precious (i.e. an expensive vase) than when it is ordinary (i.e. an old shoebox) (Almerigi, Carbary, \& Harris, 2002). It is possible that grasping a toy may not be the best action to elicit hand preference. It is also likely that handedness becomes more stable after the infant starts to master and repeat more skillful actions such as drawing, writing, eating, etc.

Another reason evoked to explain the early developmental fluctuations in hand use is change occurring in other domains, such as crawling development (Goldfield, 1989), the emergence of independent locomotion (Corbetta \& Bojczyk, 2002; Corbetta \& Thelen, 2002), babbling onset (Bates, O'Connell, Vaid, Sledge, \& Oakes, 1986; Ramsay, 1984), and the likes. If fluctuations are mainly due to change in other domains, then they should be smaller when 
the infants are retested immediately or shortly after the first evaluation compared to when they are examined weeks or months apart. In contrast, if fluctuations are due to the fact that grasping a toy is not sufficiently challenging to induce a strong hand preference, or to the fact that young infants have no strong hand preference, then, one should observe also large fluctuations when infants are tested days apart within the same age period. As opposed to the many studies evaluating the same infants at different ages (e.g. Jacobsohn et al., 2014; Nelson et al., 2013; Michel, Sheu, \& Brumley, 2002), short-term test-retest has, to our knowledge, rarely been done. One exception is Shucard and Shucard's 1990 study. In their auditory evoked-potential study, these authors observed 20 infants aged 24 to 26 weeks. The infants' handedness was tested during two (out of three) sessions within 12 days. The correlation between the scores of the two sessions was positive and significant $(r=.65)$. However, Shucard and Shucard (1990) used a single toy, a rattle, presented three times toward the right and three times toward the left. Their methodology thus differed from the methodology of many other studies that evaluated infant handedness with more than three presentations. In addition, in most studies, toys are usually presented at the midline one at a time, or when toys are presented to the side, two identical toys are presented simultaneously one in front of the left hand and one in front of the right hand to assess infants' preferred hand use (see Fagard et al., 2016, for a summary of these studies).

Another study was also aimed at checking short-term test-retest consistency in 6month-olds (McCormick \& Maurer, 1988). These authors tested 36 infants on the hand used to grasp 26 toys during three sessions one week apart. They found that the handedness index was positively correlated between the three sessions, significantly so between sessions 2 and 3. From the categorization into handedness groups (based on a $z$-value of $+/-1$ ), $25 \%$ of infants were given the same classification across all three sessions (highly consistent), $47 \%$ shifted between having a preference and being non-lateralized (moderately consistent) and $28 \%$ shifted between right and left preference over the sessions (inconsistent). The authors concluded that the infants were "more consistent than expected by chance" (McCormick \& Maurer, 1988, p. 25). Note that McCormick and Maurer calculated an index based on the difference between right and left-hand grasps divided by the square root of [right + left hand grasps], thus neglecting bimanual grasps. It has been shown that not including bimanual grasps leads to a great underestimation of the non-lateralized infants (Fagard et al., 2016).

Both of these studies tested infants around 6 months. At 6 months, infants are not yet very skillful at grasping objects, and it would be useful to know to what extent the measure of handedness is reliable after infants have become competent graspers. In the study presented here, we evaluated hand preference for grasping toys presented at the midline to the same infants within the same week (five day-to-day evaluations). We chose to test infants around 915 months of age: at that age reach to grasp is well developed, the age period includes the 10 month-olds' peak of lateralized reaching according to Michel et al. 2014' study, and in addition there are strong locomotor changes with potential consequences on infant's handedness, as mentioned above. In particular, we expected locomotor infants to show a more consistent lateralized reaching pattern than non-locomotor (Corbetta \& Bojczyk, 2002). The question we asked in this study is as follow: when we test handedness in an infant of that age on one day, would we obtain the same handedness result if we had tested him/her the next day or the day after? In addition, we investigated whether the locomotor stage of the infant would influence the consistency of handedness evaluated through test-retest reliability. These questions are important to estimate the validity of our evaluation of handedness at one point in time, which 
is the case when handedness evaluation is used as a complement to testing other neuropsychological functions potentially related to handedness.

\section{Method}

\section{Participants}

Twenty-one infants (13 boys) from three different day care centers were tested at their day care. They were $91 / 2$ to 15 -month-olds (Mean $=11.5$ months). The mean age of the girls (10.9 months) and boys (11.8 months) did not differ significantly, $p=.20$. Parental consent was granted before observing the infants. In addition to being tested for handedness, we recorded whether or not the infants crawled or walked independently. All infants crawled, five infants walked independently. We also asked parents whether they were right- or left-handed (writing hand and hand performing most daily activities). All mothers were right-handed and four fathers were left-handed.

\section{Procedure and materials}

\section{Tests of handedness}

All infants were given a classical handedness baby test with objects presented at the midline. The baby handedness test (BbHtest) comprises 11 items as a mean. One study showed that starting with 5 objects presentations, the handedness index is significantly correlated with the handedness index obtained from 34 object presentations, and that starting with 10 trials, the risk of misevaluating the direction of handedness is very low at 11 months (Fagard et al., 2016). Objects were small plastic or wooden baby toys, between 0.5 and $7 \mathrm{~cm}$ wide and between 2 and $17 \mathrm{~cm}$ high (see examples on Figure 1). We avoided objects affording bimanual manipulation, such as when one part of the object can be manipulated, since in this case, the grasping hand may anticipate stabilizing the object for the more active hand to manipulate it. The order of presentation was random. All objects were presented within reaching distance of the infant at a midline position. Infants were tested during five sessions over 5 consecutive days with a maximum of two days in between two testing sessions. Testing always took place in the same room at the infant's regular day care center.

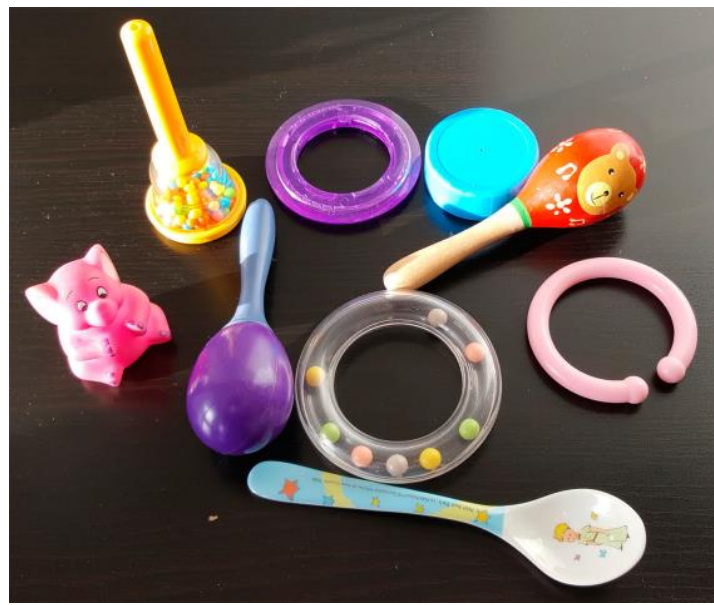

Figure 1 Examples of the objects of the baby handedness test 


\section{Analyses}

A handedness index $(\mathrm{HI})$ was calculated as follow: [(Number of $\mathrm{RH}$ grasps - Number of LH grasps) / (Number of RH grasps + Number of LH grasps + Number of Bimanual grasps)]. We included the number of bimanual grasps in the formula after finding that not including bimanual grasps leads to an underestimation of the non-lateralized infants (Fagard et al., 2016). A first analysis was performed on the overall mean handedness index $(\mathrm{HIm})$ calculated across the 5 daily testing sessions corresponding to a maximum of 84 object presentations (mean number of presentations $=56.3$ ). Infants with $\mathrm{HIm} \geq 0.30$ were considered as righthanded whereas those with HIm $\leq-0.30$ were considered as left-handed. All infants with HIm falling between these two values were considered as non-lateralized. This [0.30] cut-off to distinguish between lateralized and non-lateralized individuals was drawn from a former study of 716 individuals who were administered a handedness questionnaire. Multiple correspondence analyses were applied to the 716 questionnaires to define handedness groups, categorizing participants as either right-handed, left-handed or non-lateralized. We then compared this categorization with handedness index (HI)-based categories using different cut-offs and found that it was most correlated with $\mathrm{HI}$ cut-offs at -30 and +30 , delimiting lefthandedness, non-lateralization and right-handedness (Fagard et al., 2016).

We looked at the consistency of the handedness index across the five sessions (HId1 to HId5) by computing a $\mathrm{HI}$ per day and by categorizing those Hls using the same thresholds of $\leq+-0.30$. In addition, we considered whether non-lateralized infants had a $\mathrm{HI}<0$ or $>0$.

Because $\mathrm{HI}$ scores were not normally distributed, we applied non-parametric tests to assess differences. Friedman ANOVA was used to test change between sessions. Spearman rank correlations were calculated between individual Hls to assess day-to-day consistency. Specifically, correlations were performed (1) between Hls of each session, (2) between each $\mathrm{HI}$ and the $\mathrm{HIs}$ of the 4 other sessions, and (3) between $\mathrm{HI}$ of each session and the mean $\mathrm{HI}$ of all 5 sessions $(\mathrm{HIm})$.

\section{Results}

\section{HIm for all 5 sessions, HIs for each session: analysis at the group level}

The overall HIm was 0.32 (from -0.38 to 0.92 , SD =0.39). There was no significant difference between assessments in the three daycare centers, $p=.15$, and no difference in HIm depending on sex, $p=.85$.

Table 1 shows the Hls obtained for each daily session. There are some variations across sessions, with the $\mathrm{HI}$ tending to increase from sessions 1 to 2 and then to decrease slightly afterward. However, a Friedman ANOVA indicated that these differences across sessions were not significant, $p=.10$. 
Table 1. Group mean Hls and standard deviations as a function of sessions (HI day 1 to day 5).

\begin{tabular}{lcc}
\hline Sessions & Mean HI & SD \\
\hline d1 & 0.16 & 0.49 \\
d2 & 0.48 & 0.48 \\
d3 & 0.45 & 0.44 \\
d4 & 0.32 & 0.61 \\
d5 & 0.27 & 0.61 \\
\hline
\end{tabular}

\section{Comparing Hls from session to session}

The correlations between Hls for each of the 5 sessions (HId1 to HId5) are all positive but only the correlations between the third and the fourth, between the third and the fifth, and between the fourth and the fifth day of testing are significant (Table 2). The last two columns of the table show that correlations between Hls obtained from each individual session were positively correlated with HIm obtained from the 5 sessions (column 5 ), even when the session itself was excluded (column 6). All correlations are significant except for session 2 which does not reach significance.

Table 2. Correlations between daily HIs.

\begin{tabular}{lllllcc}
\hline$N=21$ & HI D2 & HI D3 & HI D4 & HI D5 & Hlm D12345 & All other sessions \\
\hline HI D1 & \multirow{2}{*}{0.17} & 0.26 & 0.34 & 0.43 & $0.64^{*}$ & $0.46^{*}$ \\
HI D2 & & 0.25 & 0.09 & 0.21 & 0.40 & 0.23 \\
HI D3 & & & $0.51^{*}$ & $0.79^{*}$ & $0.80^{*}$ & $0.69^{*}$ \\
HI D4 & & & & $0.53^{*}$ & $0.75^{*}$ & $0.54^{*}$ \\
HI D5 & & & & & $0.85^{*}$ & $0.72^{*}$ \\
\hline
\end{tabular}

${ }^{*}$ Correlations are significant at the .05 level.

\section{Categories from HI: consistency at the group level}

When the infants were categorized from the HIm obtained from all sessions, $57.1 \%$ were right-handed $(\mathrm{N}=12), 9.5 \%$ were left-handed $(\mathrm{N}=2)$, and $33.3 \%$ were non-lateralized $(\mathrm{N}=7)$.

To check the consistency of categorization across sessions, we first compared the categories obtained from the $\mathrm{HI}$ at each session. Figure 2 shows that, at the group level, the distributions of right-handers, left-handers and non-lateralized infants across sessions are comparable. The right-handers represent always the majority and the left-handers almost always the minority, the non-lateralized standing in between and varying between $19.1 \%$ and $33.3 \%$. The ratio of percentage between right- and left-handers varies from 2 to 7 . However, a $2 \times 2$ non-parametric McNemar test shows that the distribution of right- and left-handers never significantly varies between sessions ( $p=.19$ to .94). Thus, it seems that at the group level, there is some consistency in the distribution of handedness across the five sessions. 


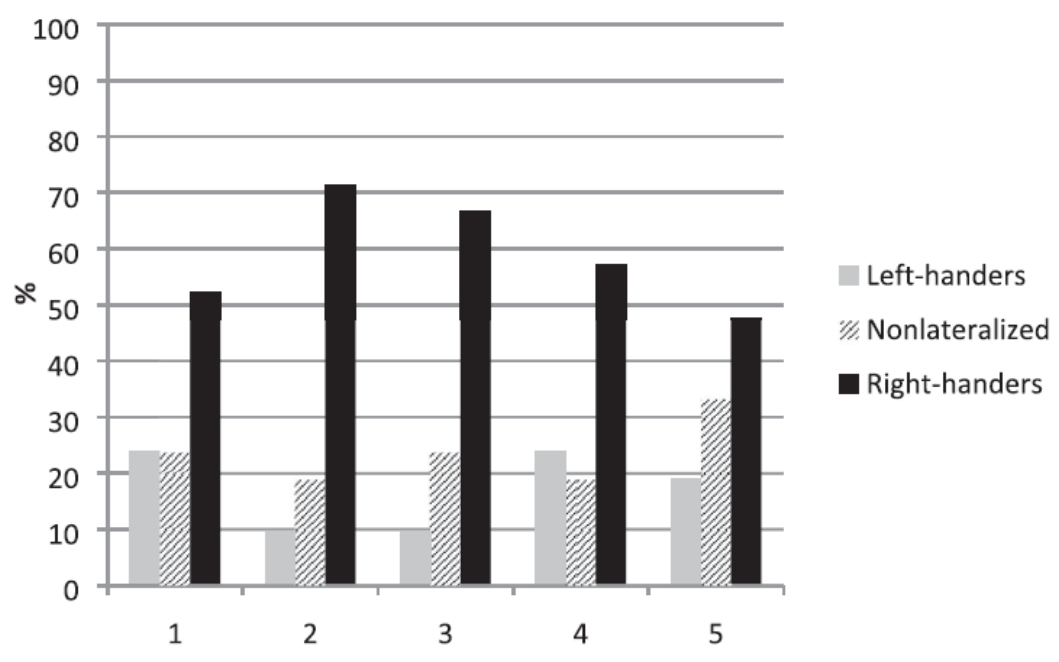

Figure 2 Distribution of handedness across the 5 sessions.

\section{Consistency of hand preference at the individual level}

Are infants always in the same category across the 5 successive sessions? In other words, is handedness measured on a given day offering a sufficiently reliable handedness measure within that age range? For this analysis, we first considered not only the category of handedness but also the value of the $\mathrm{HI}$ when infants were categorized as non-lateralized. Four infants were always categorized as right-handed across all 5 sessions (19\%) (Table 3). None of the infants were consistently left-handed or consistently non-lateralized. Some of the infants were either right-handed or non-lateralized with a positive $\mathrm{HI}$, meaning that their degree of handedness changed but not its direction. This is the case for six infants who were either categorized as right-handed at 4 sessions (except for one infant, categorized as right-handed at 2 sessions) or non-lateralized with a positive $\mathrm{HI}$. Thus 10 infants used reliably more their right hand than their left hand (47.6\%). Only one infant was consistently categorized either as left-handed (at 3 sessions) or non-lateralized with a negative $\mathrm{HI}$, meaning that its degree of handedness changed but not its direction. This resulted to 11 infants who used one hand consistently more than the other $(52.4 \%)$, seven $(33.3 \%)$ who changed category across sessions (sometimes right-handed, sometimes left-handed, other times non-lateralized), and 3 infants mostly non-lateralized who did not change category, but used more their right hand at some sessions and more their left hand at other sessions (14.3\%). Thus, 10 infants (47.6\%) changed direction of preferred hand across sessions. We can conclude that the percentage of infants who were consistent in the direction of handedness is roughly equal to the percentage of infants inconsistent in their preferred hand across sessions, and that among these consistent infants $(\mathrm{N}=11)$, only one reliably used its left hand, whereas all other 10 infants more reliably used their right hand. 
Table 3. Infants' classification for consistency of hand preference across sessions $(\mathrm{RH}=$ right-handed at the 5 sessions; $\mathrm{RH} / \mathrm{NL}=$ right-handed at most sessions and with $\mathrm{HI}>0$ when non-lateralized; $\mathrm{LH} / \mathrm{NL}=$ left-handed at most sessions and with $\mathrm{HI}<0$ when non-lateralized; $\mathrm{RH} / \mathrm{LH} / \mathrm{NL}=$ changing categories across sessions; $\mathrm{NL}=$ non-lateralized at all sessions but with $\mathrm{HI}<0$ at some sessions and $\mathrm{HI}>0$ at other sessions).

\begin{tabular}{|c|c|c|c|c|c|c|}
\hline & $N$ & $\underset{N}{\text { Cumulated }}$ & $\%$ & $\begin{array}{l}\% \text { per } \\
\text { category }\end{array}$ & Category & $\begin{array}{l}\text { Consistent vs. } \\
\text { inconsistent }\end{array}$ \\
\hline $\mathrm{RH}$ & 4 & 4 & 19 & 19 & $\begin{array}{l}\text { Consistently categorized } \\
\text { as right-handed }\end{array}$ & 1 \\
\hline $\begin{array}{l}\mathrm{RH} \text { or } \mathrm{NL} \\
\text { with } \mathrm{HI}>0\end{array}$ & 6 & 10 & 28.6 & 47.6 & $\begin{array}{l}\text { Consistent in using more } \\
\text { the right hand }\end{array}$ & \\
\hline $\begin{array}{l}\text { LH or NL with } \\
\qquad \mathrm{HI}<0\end{array}$ & 1 & 11 & 4.8 & 52.4 & $\begin{array}{l}\text { Consistent in using more } \\
\text { one hand }\end{array}$ & I \\
\hline $\mathrm{RH} / \mathrm{LH} / \mathrm{NL}$ & 7 & 18 & 33.3 & 47.6 & Inconsistent across & 2 \\
\hline $\begin{array}{r}\text { NL with } \mathrm{HI}< \\
0 \text { or } \mathrm{HI}>0\end{array}$ & 3 & 21 & 14.3 & & sessions & \\
\hline Total & & 21 & 100 & & & \\
\hline
\end{tabular}

We then considered the category of handedness stricto sensu, thus considering only the $[0,30]$ cut-off. When changes in category $(\mathrm{RH}, \mathrm{NL}, \mathrm{LH})$ from one session to the next are compared depending on whether the change occurs between being lateralized and nonlateralized (from $\mathrm{RH}$ to $\mathrm{NL}$ or from $\mathrm{LH}$ to $\mathrm{NL}$ ) or between being $\mathrm{RH}$ at some sessions and $\mathrm{LH}$ at other sessions, it is clear that most of the changes were between being lateralized and not lateralized. Infants changed between 0 and 4 times between being lateralized ( $\mathrm{RH}$ or $\mathrm{LH})$ and not lateralized $(\mathrm{NL})$, (mean $=0.95$ times, $S D=0.2$ ) and only between 0 and two times between being categorized as $\mathrm{RH}$ or $\mathrm{LH}$ (mean $=0.48$ times $\mathrm{SD}=0.16$ ).

\section{Factors influencing the consistency of hand preference across sessions}

We then checked whether the 10 infants consistent in preferring the right hand (category 1 of Table 2) differed in terms of sex, father handedness (no mother was lefthanded), and locomotion, from the 10 infants inconsistent across session (category 2 of Table 2). Boys were slightly more represented than girls in the inconsistent category ( $54 \%$ vs. $43 \%$ ), as well as non-locomotor infants versus walking infants (53.3\% vs. $40 \%)$ but none of these differences was significant. The only infant being consistent in the direction of left-handedness was one of the four infants with a left-handed father; the other left-handed fathers had an infant either inconsistent across sessions $(\mathrm{N}=1)$ or consistently right-handed $(\mathrm{N}=2)$.

\section{Consistency of category of hand preference obtained from $\mathrm{HI}$ at the first session and from Hls with repeated measures (session 2-5)}

Since the question asked was "when we test an infant one day, what confidence can we have that the results of this testing reflects validly the infant's handedness", and since using the raw $\mathrm{HI}$ may be impossible for some analyses, we compared the handedness category of the infant at the first session with the handedness category obtained from the mean $\mathrm{HI}$ calculated for all other four sessions. Since, as we saw (Table 2), HI at day 1 was significantly correlated with HIs for all other sessions, we can expect the classifications not to differ greatly. Nine infants (42.9\%) were right-handed at both classifications, two (9.5\%) were non-lateralized at both classifications, which means $52.4 \%$ of them remained in the same category. Nine infants $(42.9 \%)$ changed from being right-handed or left-handed to being non-lateralized or from being non-lateralized to being right-handed (Table 4). Only one infant (4.8\%) was 
categorized with a different direction of handedness at session 1 and at all the other sessions cumulated.

Table 4. Infants' classification at session 1 and from the mean of sessions 2-5.

\begin{tabular}{lllc}
\hline Session 2345 Session 1 & $\mathrm{RH}$ & $\mathrm{NL}$ & $\mathrm{LH}$ \\
\hline $\mathrm{RH}$ & $9(42.9 \%)$ & $2(9.5 \%)$ & 0 \\
$\mathrm{NL}$ & $2(9.5 \%)$ & $2(9.5 \%)$ & $1(4.8 \%)$ \\
LH & $1(4.8 \%)$ & $4(19 \%)$ & 0 \\
\hline
\end{tabular}

\section{Discussion}

The goal of this study was to evaluate to what extent infants' handedness tested at a single session is correlated with subsequent tests made one to five days later. The study was aimed at answering the question that most researchers should ask themselves: what is the real validity of my observation today? Would the results be the same, had I made the observation a few days later? In addition, we asked which factors might influence the consistency: sex, parents' handedness, and locomotor status.

A global analysis of infants' handedness across all 5 sessions showed a positive mean handedness index $(\mathrm{HIm})$ which indicated a tendency toward right-handedness at the group level. The percentages of right-handers, left-handers, and non-lateralized infants obtained from $\mathrm{HIm}$ (57.1\%, 9.5\%, and 33.3\%, respectively) were in line with previous findings observing a majority of right-handers but more non-lateralized infants than in adults (Corbetta \& Thelen, 1999; Fagard, 1998; Nelson, Campbell, \& Michel, 2013).

To evaluate test-retest reliability, we first analyzed the consistency between the handedness index $(\mathrm{HI})$ obtained from the five successive or almost successive days. First, even though the $\mathrm{HI}$ varied slightly from session to session, the differences across sessions were not significant. Then, the correlations between all $5 \mathrm{HIs}$ were all positive, some of them reaching significance, especially during the last 3 sessions. This result is in line with Shucard and Shucard's 1990 study who found that the handedness score of 24 to 26 -week-old infants tested 12 days apart was significantly correlated $(r=.65)$.

When the categories derived from the $\mathrm{HI}$ were considered (with the [0.30] cut-off), at the group level, the relative frequencies of right-handers, left-handers and non-lateralized infants did not change tremendously across sessions despite slight variations. At most sessions there was a majority of right-handers, a minority of left-handers and, in between, a larger percentage of non-lateralized than in adults. The ratio between right- and left-handers did not change significantly across sessions.

However, the comparable distribution of handedness categories at the group level does not mean that the same infants remain in the same category from one session to the next. Slightly less than half of the infants in our sample (47.6\%) could be considered as having a consistent preference for the right-hand across sessions (being right-handed at most sessions, never left-handed, and always having a $\mathrm{HI}>0$ when non-lateralized). Among them, $19 \%$ were consistently categorized as right-handed across all five sessions. Only one infant $(4.8 \%)$ showed consistent preference for her left hand, being left-handed at most sessions, never right-handed, and always having a $\mathrm{HI}<0$ when non-lateralized. Thus, slightly more than half 
of the infants were consistent in their hand preference (52.4\%), without being always consistent in the degree of their hand preference. Note that none of the infants were categorized as lefthanded at each of the five sessions. Finally, $47.6 \%$ of the infants were inconsistent not only in the degree of handedness but also in its direction, shifting direction of handedness at least once when lateralized, or having sometimes a $\mathrm{HI}<0$ and other times a $\mathrm{HI}>0$ when nonlateralized. The percentage of infants (19\%) who were right-handed at all five sessions was lower than the $30-35 \%$ reported by Nelson et al. (2014) for consistent right-handedness from the first evaluation and across 6-14 months. But if we add the infants who were either righthanded or had a $\mathrm{HI}>0(47.6 \%)$, then one could say that almost the majority of them at that age were consistent in showing a preferred bias for the right-hand. Counting the infant with a left-hand preference, more than half of the sample was consistent in the direction of their hand preference. However, the same could not be said for the degree of hand preference: most changes in category from session to session involved changing from being lateralized to nonlateralized (or vice versa).

Finally, when the category obtained from $\mathrm{HI}$ at the first session was compared to the categories obtained from the mean of all other sessions, only one infant was LH at the first session and $\mathrm{RH}$ at some sessions. More than half of the infants were in the same category but $42.8 \%$ were categorized as lateralized at the first session ( $\mathrm{RH}$ or $\mathrm{LH}$ ) and non-lateralized (NL) from the mean of all other sessions (or vice versa). This indicates that testing at one session can lead to an underestimation or overestimation of the degree of handedness but rarely to an error in the direction of handedness.

None of the factors investigated (sex, parents' handedness, and locomotion) influenced significantly the consistency of handedness across sessions.-Boys were non-significantly more represented than girls in the inconsistent category. Non-locomotor infants were also nonsignificantly more represented in the inconsistent category than the walking infants. This tendency is interesting to relate to the observation that after infants gain better balance for walking, there is a sharp increase in right-handedness (Corbetta, Friedman, \& Bell, 2014). Finally, the only consistently left-handed infant was the daughter of one of the four left-handed fathers.

In conclusion, these results show that when we measure infants' hand preference at the age period considered, one can obtain rather reliable results when direction is being considered and when testing is repeated within a week. As long as the assessment tool is valid and the method used appropriate, testing the infant at a single session can be considered a valid reflection of the infant's direction of hand preference but less so of its degree. For this reason, it is preferable to consider the $\mathrm{HI}$ rather than the category obtained from the $\mathrm{HI}$. 


\section{References}

Almerigi, J. B., Carbary, T. J., \& Harris, L. J. (2002). Most adults show opposite-side biases in the imagined holding of infants and objects. Brain Cogn, 48(2-3), 258-263.

Bates, E., O'Connell, B., Vaid, J., Sledge, P., \& Oakes, L. (1986). Language and hand preference in early development. Developmental Neuropsychology, 2(1), 1-15.

Bryden, P. J., \& Roy, E. A. (2006). Preferential reaching across regions of hemispace in adults and children. Dev Psychobiol, 48(2), 121-132.

Campbell, J. M., Marcinowski, E. C., Latta, J., \& Michel, G. F. (2015). Different assessment tasks produce different estimates of handedness stability during the eight to 14 month age period. Infant behavior and Development, 39, 67-80.

Corbetta, D., \& Thelen, E. (1999). Lateral biases and fluctuations in infants' spontaneous arm movements and reaching. Developmental Psychobiology, 34(4), 237-255.

Corbetta, D., \& Bojczyk, K. E. (2002). Infants return to two-handed reaching when they are learning to walk. J Mot Behav, 34(1), 83-95.

Corbetta, D., Friedman ${ }^{\dagger}$, D. R., \& Bell, M. A. (2014). Brain reorganization as a function of walking experience in 12 month-old infants: Implications for the development of manual laterality. Frontiers in Psychology: Cognition, 5:245. DOI: 10.3389/fpsyg.2014.00245.Corbetta, D., \& Thelen, E. (1999). Lateral biases and fluctuations in infants' spontaneous arm movements and reaching. Developmental Psychobiology, 34(4), 237-255.

Corbetta, D., \& Thelen, E. (2002). Behavioral fluctuations and the development of manual asymmetries in infancy: Contribution of the dynamic systems approach. In S. J. Segalowitz \& I. Rapin (Eds.), Handbook of Neuropsychology (Vol. 8): Child Neuropsychology, Part I (pp. 309-328). Amsterdam, the Netherlands: Elsevier Science Publishing Co.

de Vries, J. I. P., Wimmers, R. H., Ververs, I. A. P., Hopkins, B., Savelsbergh, G. J. P., \& van Geijn, H. P. (2001). Fetal handedness and head position preference: A developmental study. Developmental Psychobiology, 39(3), 171-178.

Fagard, J. (1998). Changes in grasping skills and the emergence of bimanual coordination during the first year of life. In K. J. Connolly (Ed.), The Psychobiology of the hand (Vol. Clinics in Developmental Medicine, pp. 123-143). Londres: Mac Keith Press.

Fagard, J., \& Lockman, J. J. (2005). The effect of task constraints on infants' (bi)manual strategy for grasping and exploring objects. Infant Behavior \& Development, 28(3), 305315.

Fagard, J., Margules, S., Lopez, C., Granjon, L., \& Huet, V. (2016). How should we test infant handedness? Laterality, 22(3), 294-312. doi: 0.1080/1357650X.2016.1192186.

Gabbard, C., \& Helbig, C. R. (2004). What drives children's limb selection for reaching in hemispace? Exp Brain Res, 156(3), 325-332.

Gabbard, C., \& Rabb, C. (2000). What determines choice of limb for unimanual reaching movements? Journal of General Psychology, 127(2), 178-184.

Gabbard, C., Rabb Helbig, C., \& Gentry, V. (2001). Lateralized effects on reaching by children. Developmental Neuropsychology, 19(1), 41-51.

Gesell, A., \& Ames, L. B. (1947). The development of handedness. Journal of Genetic Psychology, 70, 155-175.

Goldfield, E. C. (1989). Transition from rocking to crawling: postural constraints on infant movement. Developmental Psychology, 25, 913-919.

Hepper, P. G., Shahidullah, S., \& White, R. (1991). Handedness in the human fetus. Neuropsychologia, 29(11), 1107-1111.

Hepper, P. G., Wells, D. L., \& Lynch, C. (2005). Prenatal thumb sucking is related to postnatal handedness. Neuropsychologia, 43(3), 313-315.

Jacobsohn, L., Rodrigues, P., Vasconcelos, O., Corbetta, D., \& Barreiros, J. (2014). Lateral manual asymmetries: A longitudinal study from birth to 24 months. Developmental Psychobiology, 56, 58-72. DOI: 10.1002/dev.21091. 
Leconte, P., \& Fagard, J. (2004). Influence of object spatial location and task complexity on children's use of their preferred hand depending on their handedness consistency. Dev Psychobiol, 45(2), 51-58.

Leconte, P., \& Fagard, J. (2006). Which factors affect hand selection in children's grasping in hemispace? Combined effects of task demand and motor dominance. Brain Cogn, 60(1), 88-93.

McCormick, C. M., \& Maurer, D. M. (1988). Unimanual hand preferences in 6-month-olds: Consistency and relation to familial-handedness. Infant Behavior and Development, 11(1), 21-29.

McManus, C. (2002). Right Hand, Left Hand: The Origins of Asymmetry in Brains, Bodies, Atoms and Cultures. London: Weindenfeld \& Nicolson.

Michel, G. F., Sheu, C. F., \& Brumley, M. R. (2002). Evidence of a right-shift factor affecting infant hand-use preferences from 7 to 11 months of age as revealed by latent class analysis. Dev Psychobiol, 40(1), 1-13.

Michel, G. F., Babik, I., Sheu, C. F., \& Campbell, J. M. (2014). Latent classes in the developmental trajectories of infant handedness. Dev Psychol, 50(2), 349-359. doi: 2013-21353-001 [pii] 10.1037/a0033312.

Nelson, E. L., Campbell, J. M., \& Michel, G. F. (2013). Unimanual to bimanual: Tracking the development of handedness from 6 to 24 months. Infant Behav Dev, 36(2), 181-188.

Nelson, E. L., Campbell, J. M., \& Michel, G. F. (2014). Early handedness in infancy predicts language ability in toddlers. Dev Psychol, 50(3), 809-814.

Ramsay, D. S. (1984). Onset of duplicared syllable babbling and unimanual handedness in infancy: evidence for developmental change in hemispheric specialization? Developmental Psychology, 20, 64-71.

Shucard, J. L., \& Shucard, D. W. (1990). Auditory evoked potentials and hand preference in 6Month-Old Infants: possible gender-related differences in cerebral organization. Developmental Psychology, 26, 923-930. 


\section{Captions}

Figure 1: Distribution of handedness across the 5 sessions

Table 1: Group mean HIs and standard deviations as a function of sessions ( $\mathrm{HI}$ day 1 to day 5 )

Table 2: Correlations between daily HIs. Correlations in red are significant at the .05 level

Table 3: Infants' classification for consistency of hand preference across sessions ( $\mathrm{RH}=$ right-handed at the 5 sessions; $\mathrm{RH} / \mathrm{NL}=$ right-handed at most sessions and with $\mathrm{HI}>0$ when non-lateralized; $\mathrm{LH}$ / $\mathrm{NL}=$ left-handed at most sessions and with $\mathrm{HI}<0$ when non-lateralized; $\mathrm{RH} / \mathrm{LH} / \mathrm{NL}=$ changing categories across sessions; $\mathrm{NL}=$ non-lateralized at all sessions but with $\mathrm{HI}<0$ at some sessions and $\mathrm{HI}>0$ at other sessions

Table 4: Infants' classification at session 1 and from the mean of sessions 2 to $5(\mathrm{RH} / \mathrm{RH}=$ right-handed at the first and at sessions 2-5; NL / NL at the first and at sessions 2-5 = non-lateralized; $\mathrm{RH} / \mathrm{NL}$ or NL / $\mathrm{RH}=$ right-handed at session 1 and non-lateralized at sessions 2 to 5 or vice versa; $\mathrm{LH} / \mathrm{NL}=$ lefthanded at session 1 and non-lateralized at sessions 2 to 5 ; $\mathrm{LH} / \mathrm{RH}=$ left-handed at session 1 and righthanded at sessions 2 to 5 ) 